in Wissentransfer 2, p.84, TU Berlin (1996)

\title{
Hydrodynamic Modes of Viscoelastic Polymer Films
}

\author{
HARALD PLEINER \\ Max-Planck-Institut für Polymerforschung, Postfach 3148, 55021 Mainz
}

\section{Introduction}

Hydrodynamics of simple fluids (liquids and gases) is a classical textbook subject that still bears a lot of interesting and unsolved problems (e.g. turbulence) due to its inherent nonlinear nature. The use of computers and the renewed interest in nonlinear phenomena (e.g. instabilities, pattern formation) has led to a revival of that classical subject. On the other hand it has become possible to apply hydrodynamics also to more complex systems. This was facilitated by a deeper understanding of hydrodynamics based on symmetries and thermodynamics [1-4]. It can now be used as a general method to describe macroscopically the dynamics of many condensed systems including liquid crystals, superfluid liquids, crystals, magnetic systems etc. The foundations for the linear hydrodynamic description of liquid crystals were laid in the '70s [5-8], while in the '80s this method was further applied to nonlinear descriptions $[9,10]$ and to increasingly more complex liquid crystal phases [11-16].

The hydrodynamic method is based on the observation that in most condensed systems there is only a small number of slow, long living processes compared to the huge number of fast, microscopic degrees of freedom, which can be discarded in a description of the macroscopic behaviour. The hydrodynamic variables describe cooperative phenomena that do not relax in finite time in the homogeneous limit, i.e. for the Fourier transformed modes the frequency $\omega$ vanishes for vanishing wavevector $k, \omega(k \rightarrow 0) \rightarrow 0$. The point is that these hydrodynamic variables can uniquely be identified using conservation laws (related to global symmetries) and broken symmetries (in the case of complex systems). For time and length scales, on which all the fast, local microscopic degrees of freedom have relaxed to their equilibrium value, this hydrodynamic description is exact.

In some cases a few of the non-hydrodynamic, relaxing processes become so slow that their relaxation time is comparable to hydrodynamic time scales [17-18]. Examples are elastic stress relaxation in polymers, order parameter relaxation near second order (or weakly first order) phase transitions (giving rise to soft modes), relative rotations of nematic side chains with respect to the backbone segments in side-chain polymers etc. A strictly hydrodynamic theory (considering only hydrodynamic variables) would be confined in its applicability to times longer than such slow relaxation times and thus, be insufficient for many purposes. In that situation it is reasonable to extend hydrodynamics to "macroscopic dynamics" incorporating the slow relaxational variables. The first example of this procedure was used to describe the (mean field) dynamics of a superfluid near the phase transition to the normal fluid [3] by including one soft mode (the order parameter strength). Similarly slowly relaxing variables are also important for the dynamics near various phase transitions in liquid crystals [19-29] and near liquid 
crystalline defects, although in the latter case generally they are only used quasistatically [30-35]. However, there are no general rules or arguments, when or what kind of slowly relaxing variables exist in a given system. For polymers [36-38] this will be discussed in Sec. 2

Since the microscopic degrees of freedom have reached their thermodynamic equilibrium state ("local thermodynamic equilibrium") on the hydrodynamic time scale, one can use thermodynamics (locally) to describe the remaining slow variables. This leads immediately to the energetics of the system, i.e. a thermodynamic potential (e.g. the energy) as a function of all variables, or equivalently the thermodynamic conjugate quantities expressed by the variables using phenomenological static susceptibilities (e.g. the density expressed by the pressure via the compressibility). In a second step the dynamics of the system is obtained by expressing the currents or quasi-currents by the thermodynamic forces (the gradients of the thermodynamic conjugates). These expansions contain dynamic phenomenological coefficients (transport parameters). Within the thermodynamic framework it is very fruitful to split up the currents and quasi-currents into additive reversible (entropy conserving) and irreversible (entropy increasing) parts. This procedure is explained in more detail in [39].

In order to derive a hydrodynamic or macroscopic description of thin films or interfaces made of complex fluids, one can setup the appropriate three-dimensional theory and then take the limit of vanishing film thickness. For the systems we have in mind insoluble polymers, wetting layers of entangled polymers, polymeric surfactant films, layers of polymeric liquid crystalline material etc. between or on top of bulk liquids this procedure leads to an effective bulk problem, where the dynamic properties of the film or interface are manifest in complex boundary conditions. Very often such effective boundary conditions are postulated or assumed, while the present treatment allows a systematic derivation and a detailed connection to known bulk properties of the film or interfacial material.

The main advantage of the hydrodynamic method rests in its high generality, which allows its application to very different systems. There are no model dependent assumptions and only very fundamental symmetry and thermodynamic arguments are used. The occurence of phenomenological parameters in the static and dynamic expansions, however, are the prize one has to pay for this generality. The only restriction on the applicability of a hydrodynamic theory arises from the validity of the static and dynamic expansions used. Going beyond hydrodynamics it is not possible to predict, if and which non-hydrodynamic variables can become slow, although the generalized theory, which includes such variables, is still a powerful theory albeit less fundamental than a purely hydrodynamic theory. Its practical use is confined to situations with a clear-cut distinction between the macroscopic time scale (relevant for only a few variables) and the microscopic one (relevant for all the others)

\section{Hydrodynamic Film Equations}

For the three-phase system we are going to describe, bulk fluid ${ }_{1} /$ interface / bulk fluid ${ }_{2}$, we have to write down hydrodynamic equations for each phase separately. For simplicity we will assume the bulk fluids to be simple liquids, although a generalization to complex liquids would be easy at that point. Thus, the bulk is described by two different sets 
of usual hydrodynamic equations, which includes the popular special cases of symmetric fluids ( liquid $_{1}$ equal to liquid 2 ) or surface layers (liquid 2 equal to vapor or vacuum).

The interface or film shall be a polymeric fluid. Polymer solutions or melts differ from simple (low molecular weight) liquids by their viscoelasticity. On short time and length scales they sustain elastic stresses, while in the low frequency, long wavelength limit they flow like a usual viscous fluid. This behaviour can be accounted for by introducing a slowly relaxing field, which describes the transient elasticity. Similarly to crystals and solids, whose permanent elasticity is described by a second rank strain tensor, we also use a symmetric second rank tensor as macroscopic variable in the polymeric case. In crystalline material, however, strains are related to displacements of particles, which return to their original position in equilibrium and the strain tensor is derived from a displacement vector (taking symmetrized gradients of it). This displacement vector is the symmetry variable of crystals connected with the spontaneous breaking of translational symmetry due to the appearance of a lattice. In polymers on the other hand, no such equilibrium lattice exists and strains are not related to displacements, but to dynamical entanglements of long chains. Thus we regard the slowly relaxing strain tensor in polymers as containing six independent macroscopic variables, three of which transform into symmetry variables and three into microscopic variables, when switching to permanent elasticity [40]. In the linear domain this model is equivalent to a generalized Maxwell approach, although taking the polymeric strain explicitly as a dynamical variable (instead of using complex and frequency dependent transport parameters in an ad hoc manner), allows the necessary generalizations to liquid crystalline or otherwise complex systems. Especially the coupling to other macroscopic variables is straightforward in the present approach, but hardly feasible in the generalized Maxwell description. Shear thinning or thickening effects can be dealt with in principle by allowing the viscosities to depend on the dynamic variables, while normal stress effects are not covered.

The internal, macroscopic state of the film or membrane is described by the fields $\rho_{m}$ (density), $g_{i}^{(m)}$ (momentum density), $\epsilon_{m}$ (energy density) and $\epsilon_{i j}$ (transient elastic strain). Since we will take the limit of vanishing film thickness at the end, it is sufficient to take the fields at the mid-plane of the film, e.g. $\rho_{m}=\left.\rho\right|_{z=0}$. Thus all fields are twodimensional (areal) densities instead of three-dimensional (volume) densities. Since we are interested in small deviations of the film from its flat equilibrium state, the mid-plane can be taken as $z=0$. Changes of the variables are connected to entropy changes $(d s)$ by the Gibbs relation

$$
T d s=d \epsilon_{m}-\mu d \rho_{m}-v_{i} d g_{i}^{(m)}-\psi_{i j} d \epsilon_{i j},
$$

thus defining the conjugate quantities temperature $T$, chemical potential $\mu$, velocity $v_{i}$ and transient elastic stress $\psi_{i j}$. There are two conservation laws for mass and momentum

$$
\dot{\rho}_{m}+\nabla_{i} g_{i}^{(m)}=0 \quad \text { and } \quad \dot{g}_{i}^{(m)}=\sigma_{i j}^{(m)}=0,
$$

where $\sigma_{i j}^{(m)}$ is the stress tensor of the film. Using the entropy (instead of the energy) as dynamical variable there are two generally non-conserved quantities

$$
\dot{s}+\nabla_{i} j_{i}=\frac{R}{T} \quad \text { and } \quad \dot{\epsilon}_{i j}+X_{i j}=0
$$


with $j_{i}$ the entropy current density and $R$ the dissipation function. According to the laws of thermodynamics $R$ is zero for reversible processes (and the entropy conserved), while dissipative dynamics leads to $R>0$. The quasi-current $X_{i j}$ just reflects the relaxative dynamics of the transient elasticity.

To get a closed system of equations we have to express the currents by the variables. This is a two-stage process comprising the static and the dynamic constitutive equations. First the conjugate quantities, defined in (1), are expanded into the variables (and into their gradients if symmetry variables are involved) using general symmetry and invariance principles. The most efficient way to obtain this is to setup a phenomenological energy functional, which defines the conjugate quantities via variational derivation. In the present case we have

$$
\epsilon_{m}=\epsilon_{0}+\frac{c_{k}}{2} \epsilon_{\alpha \alpha}^{2}+c_{s}\left(\epsilon_{\alpha \beta}-\frac{1}{2} \epsilon_{\gamma \gamma} \delta_{\alpha \beta}\right)^{2}+c_{\perp} \epsilon_{\alpha z}^{2}+c_{b}\left(\nabla_{\perp} \epsilon_{\alpha z}\right)^{2}
$$

where $\{\alpha, \beta, \gamma\} \in\{x, y\}$. In (4) $\epsilon_{0}$ denotes the usual free energy of a simple liquid containing the specific heat, the compressibility $\chi$ and the thermal expansion coefficient as static susceptibilities. The elastic constants $c_{k}, c_{s}, c_{\perp}$ and the (orientational elastic constant) $c_{b}$ describe in-plane compression, in-plane shear, transverse stretching and bend of the film, respectively. Although the material of the film is assumed to be isotropic, the film has a preferred direction rendering it uniaxial and leading to $c_{s} \neq c_{\perp}$. The bend, which is neglected in bulk elasticity, can be important for films or membranes. Film compression, $\epsilon_{z z}$ does not occur in thin films. Nonlinearities could be incorporated by going beyond the bilinear forms in (4).

The currents and quasi-currents are split into two parts each, a reversible and an irreversible one. Both sets are then expanded into the thermodynamic forces, i.e. the gradients of the thermodynamic conjugates, where the reversible and irreversible parts have to be time-reversal symmetric with $R=0$ and antisymmetric with $R>0$, respectively. For the former we find

$$
\begin{aligned}
g_{i}^{(m)} & =\rho v_{i}, & X_{i j} & =-\frac{1}{2}\left(\nabla_{i} v_{j}+\nabla_{j} v_{i}\right) \\
j_{i} & =s v_{i}, & \sigma_{i j}^{(m)} & =-v_{i} v_{j}-p \delta_{i j}+\psi_{i j}
\end{aligned}
$$

Besides the usual transport terms and the hydrostatic pressure, eq.(5) contains the elastic stress $\psi_{i j}$. The irreversible part of the currents and quasi-currents can be obtained from a phenomenological expression of the dissipation function

$$
2 R=\nu_{i j k l}\left(\nabla_{i} v_{j}\right)\left(\nabla_{k} v_{l}\right)+\nu_{b}\left(\nabla_{\alpha} \nabla_{\beta} v_{z}\right)^{2}+F_{i j k l} \psi_{i j} \psi_{k l}+G_{i j k l m n}\left(\nabla_{m} \psi_{i j}\right)\left(\nabla_{n} \psi_{k l}\right),
$$

where the tensors $\nu$ and $F$ are of the form

$$
\nu_{i j k l}=\nu_{\|} \delta_{i \alpha} \delta_{j \alpha} \delta_{k \beta} \delta_{l \beta}+\nu_{s} \delta_{i \alpha} \delta_{k \alpha} \delta_{j \beta} \delta_{l \beta}+\nu_{\perp} \delta_{i \alpha} \delta_{k \alpha} \delta_{j z} \delta_{l z}
$$

Here $\nu_{\|}, \nu_{s}$ and $\nu_{\perp}$ are the viscosities according to uniaxial in-plane compressional flow, in-plane shear flow and transverse film-stretching flow, while $\nu_{b}$ is the dissipative analogue 
to the bend elastic constant. Generally there is $\nu_{s} \neq \nu_{\perp}$ because of surface effects. The coefficients $F_{\|}, F_{s}$ and $F_{\perp}$ describe the relaxation of in-plane compressional strain, inplane shear strain and transverse film-stretching strain. If these $F$-coefficients were all zero, the elasticity would be permanent describing a solid. The tensor $G$ is therefore identified as the vacancy or defect diffusion tensor present in any solid [5] (but neglected in the usual elasticity theory). Having non-zero relaxation coefficients $F$, however, vacancy diffusion can be neglected. The positivity of the entropy production is achieved by posing some restrictions on the dissipative transport parameters $\nu, F$ and $G$. The irreversible parts of the currents and quasi-currents are derived from eq.(6) by partial derivatives with respect to the appropriate conjugate quantities, e.g. $X_{i j}=\delta R / \delta \psi_{i j}-\nabla_{k}\left(\delta R / \delta \nabla_{k} \psi_{i j}\right)$.

\section{Effective Boundary Value Problem}

We are now able to formulate the original three-phase problem as a two-phase problem with effective boundary conditions. First we start with a couple of simplifications. Since we are interested in the surface-specific hydrodynamic modes only, all equations can be linearized and incompressibility of the bulk liquids (but not of the film) can be assumed. In addition the thermal degree of freedom will be neglected. The bulk equations then read [41]

$$
\rho^{(a)} \dot{v}_{i}^{(a)}=-\nabla_{i} p^{(a)}+\eta^{(a)} \Delta v_{i}^{(a)}+\nabla_{j} \Sigma_{i j}^{(a)} \quad \text { and } \quad \operatorname{div} \mathbf{v}^{(a)}=0
$$

where $p^{(a)}$ is the pressure in each liquid $(a \in\{1,2\})$ including the gravitational contribution $\rho^{(a)} g z$ due to a gravitational field along the film normal. In Eq.(8) $\Sigma_{i j}^{(a)}$ are the components of the fluctuating stress tensor in each liquid, which are added in order to calculate the dynamic structure factor by standard procedures afterwards. Near equilibrium the fluctuating stress tensor is a Gaussian white noise process, whose second moment is related to the dissipation by [42]

$$
<\Sigma_{i j}^{(a)}(\mathbf{r}, t) \Sigma_{k l}^{(b)}\left(\mathbf{r}^{\prime}, t^{\prime}\right)>=2 \pi k_{B} T \eta^{(a)}\left(\delta_{i k} \delta_{j l}+\delta_{i l} \delta_{j k}\right) \delta_{a b} \delta\left(\mathbf{r}-\mathbf{r}^{\prime}\right) \delta\left(t-t^{\prime}\right)
$$

At the boundary between two liquids that do not penetrate, the velocity components across the boundary have to be the same in each liquid, otherwise there would be no well-defined boundary at all. In the limit of vanishing film thickness this leads to the kinematic boundary conditions

$$
v_{z}^{(1)}(x, y, z=0, t)=v_{z}(x, y, t)=v_{z}^{(2)}(x, y, z=0, t)
$$

where the boundary can be taken as the plane $z=0$, because of the linearization used. If there is no film at the interface, the boundary condition concerning the stress equilibrium reads [41]

$$
\sigma_{i z}^{(1)}(x, y, 0, t)-\sigma_{i z}^{(2)}(x, y, 0, t)=\delta_{i z} \lambda \Delta_{\perp} \zeta(x, y, t)
$$

indicating that shear stresses $(i \in\{x, y\})$ are continuous at the boundary, while the compressional stress $\sigma_{z z}$ makes a jump across the boundary due to the surface tension. 
Bending the boundary (described by the transverse Laplacian of the boundary displacement function $\zeta(x, y, t)$ ) leads to the Laplacian force (per unit area) on the r.h.s. of (11). With the film present at the interface Eq.(11) has to be amended by the force (per unit area) that can be sustained or maintained by the film. The latter is given by $\nabla_{j} \sigma_{i j}^{(m)}$ and Eq.(11) finally changes into

$$
\sigma_{i z}^{(1)}(x, y, z=0, t)-\sigma_{i z}^{(2)}(x, y, z=0, t)=\nabla_{j} \sigma_{i j}^{(m)}(x, y, t)+\delta_{i z} \lambda \Delta_{\perp} \zeta(x, y, t)
$$

The system of equations becomes closed by the geometric relation

$$
\dot{\zeta}(x, y, t)=v_{z}(x, y, t)
$$

Note that $\nabla_{i} \zeta$ is not equal to $\epsilon_{i z}^{(m)}$, because of the relaxation (and diffusion) of the latter quantity.

Due to the linearization all film quantities can be expressed by $v_{z}$, which itself is expressed by the bulk velocities via (10). Thus, the boundary conditions are completely formulated in bulk quantities arriving at a two-phase description with effective boundary conditions. In time-Fourier transformed form they read

$$
\begin{aligned}
& {\left[\eta^{(1)}\left(\nabla_{z} v_{i}^{(1)}+\nabla_{i} v_{z}^{(1)}\right)+\Sigma_{i z}^{(1)}-p^{(1)}\right]_{z=0}} \\
& -\left[\eta^{(2)}\left(\nabla_{z} v_{i}^{(2)}+\nabla_{i} v_{z}^{(2)}\right)+\Sigma_{i z}^{(2)}-p^{(2)}\right]_{z=0}=\frac{1}{i \omega}\left(C_{i} \Delta_{\perp}+\delta_{i z} D \Delta_{\perp}^{2}\right) v_{z}^{(1)}(x, y, \omega)
\end{aligned}
$$

with

$$
\begin{aligned}
C_{x}(\omega) & =\chi+i \omega \nu_{\|}+\frac{i \omega \tau_{\|} c_{\|}}{1+i \omega \tau_{\|}} \\
C_{y}(\omega) & =i \omega \nu_{s}+\frac{i \omega \tau_{s} c_{s}}{1+i \omega \tau_{s}} \\
C_{z}(\omega) & =\lambda+i \omega \nu_{\perp}+\frac{i \omega \tau_{\perp} c_{\perp}}{1+i \omega \tau_{\perp}} \\
D(\omega) & =-i \omega \nu_{b}-\frac{i \omega \tau_{\perp} c_{b}}{1+i \omega \tau_{\perp}}
\end{aligned}
$$

Eq.(14) contains the effects of in-plane compressibility $\chi$, bare surface tension $\lambda$, film viscosities $\nu_{\{\perp, s, \|\}}$, elastic $c_{b}$ and viscous $\nu_{b}$ bend coefficient, as well as the elastic $c_{\{\perp, s, \|\}}$ and relaxation coefficients of the film. The latter have been replaced by relaxation times $\tau$ with $\tau_{\{\perp, s, \|\}}^{-1}=F_{\{\perp, s, \|\}} c_{\{\perp, s, \|\}}$. Boundary conditions of the form (14) have already been given in the literature [43-45] using phenomenological ansaetze or models for the $C_{i}$ parameters. The advantage of the present method is that these parameters are derived from macroscopic-hydrodynamic equations, where the material parameters of the film have a well-defined meaning. The method can also be generalized to different kinds of films or interfaces, just by replacing the set of macroscopic equations for polymers (1-7) with a different one. If the bulk fluids are not simple ones, Eq.(8) has to be changed accordingly. 


\section{Results}

For surface-related modes the ansatz $v_{z}^{(i)} \sim \exp (i k x) \exp \left(-q^{(i)} z\right)$ translates the boundary value problem into an algebraic one, which can be solved by standard means. After some straightforward, but involved linear algebra the mode spectrum and the dynamic structure factor for surface waves is obtained. This has been done in [46] and will not be repeated here. Only a few results will be quoted. The dynamic structure factor can directly be measured in light scattering experiments [47-49].

Due to the uniaxial symmetry (in-plane isotropy) of the interface the in-plane shear modes (characterized by $C_{y}, v_{y}$ ) are decoupled from the rest. This mode does not shift or undulate the interface and is not detectable by scattering techniques. The two other modes, in-plane compression and transverse mode (characterized by $C_{x}$ and $C_{z}$, respectively), although generally coupled are decoupled in the symmetric case, fluid $d_{1} \equiv$ fluid $_{2}$. Then the transverse stretching modulus of the film augments the surface tension at finite frequencies and provides an extra source of dissipation. As a result, the peak of the dynamic structure factor of the transverse mode shifts to higher frequencies with increasing $k$ somewhat faster than the usual $k^{3 / 2}$ law. Also the peak width is anomalously broadened at intermediate $k$.

For a viscoelastic film at a liquid-vapor interface (i.e. $\rho_{2}=0=\eta_{2}$ ) the transverse and the in-plane compressional modes are coupled. In the conventional Lucassen picture [43] of monolayer hydrodynamics, this coupling is manifest as a broadening of the peak in the transverse-mode dynamic structure factor, which is maximized at some in-plane compressibility. However, in the present case the complex, frequency-dependent contribution to the in-plane compressional modulus due to entanglements significantly alters the apparent maximizing value of the compressibility. Thus interfacial viscoelasticity complicates considerably the interpretation of dynamic light scattering data.

\section{Acknowledgement}

It is a pleasure to thank H.R. Brand for the longstanding collaboration on hydrodynamics of complex fluids and J.L. Harden for our joint work on surface waves.

\section{References}

1 L.P. Kadanoff and P.C. Martin, Ann.Phys. 24, 419 (1963).

2 P. Hohenberg and P.C. Martin, Ann.Phys. 34, 291 (1965).

3 I.M. Khalatnikov, Introduction to the Theory of Superfluidity, Benjamin, New York (1965).

4 N.N. Bogoljubov, Phys.Abhandl.SU 6, 229 (1962).

5 P.C. Martin, O. Parodi and P. Pershan, Phys.Rev. A6, 2401 (1972).

6 D. Forster, Hydrodynamic Fluctuations, Broken Symmetry and Correlation Functions, Benjamin, Reading, Mass. (1975).

7 T.C. Lubensky, Phys.Rev. A6, 452 (1972).

8 M.J. Stephen and J.P Straley, Rev.Mod.Phys. 46, 617 (1974).

9 H.R. Brand and H. Pleiner, J.Phys.(Paris) 41, 553 (1980).

10 H. Pleiner and H.R. Brand, Phys.Rev. A25, 995 (1982).

11 H.R. Brand and H. Pleiner, Phys.Rev. A24, 1783 (1981) and A26, 1783 (1982), 
A30, 1548 (1984).

12 W.M. Saslow, Phys.Rev. A25, 3350 (1982).

13 M. Liu, Phys.Rev. A24, 2720 (1981).

14 H.R. Brand and H. Pleiner, J.Phys.(Paris) 45, 563 (1984).

15 H. Pleiner and H.R. Brand, Phys.Rev. A29, 911 (1984) and H. Pleiner, Mol.Cryst. Liq.Cryst. 114, 103 (1984).

16 H.R. Brand and H. Pleiner, J.Phys.II 1, 1455 (1991).

17 H. Pleiner in Incommensurate Crystals, Liquid Crystals, and Quasicrystals, eds. J.F. Scott and N.A. Clark, Plenum, New York, p. 241 (1987).

18 H. Pleiner and H.R. Brand, Europhys.Lett. 15, 393 (1991).

19 M. Liu, Phys.Rev. A19, 2090 (1979).

20 K.A. Hossain, J. Swift, J.-H. Chen and T.C. Lubensky, Phys.Rev. B19, 432 (1979).

21 B.S. Andereck and J. Swift, Phys.Rev. A25, 1084.

22 H.R. Brand and H. Pleiner, J.Phys.(Paris) 43, 853 (1982).

23 H.R. Brand and P. Bak, Phys.Rev. A27, 1062 (1983).

24 H.R. Brand and J. Swift, J.Phys.Lett.(Paris) 44, 333 (1983).

25 H.R. Brand, Mol.Cryst.Liq.Cryst.Lett. 4, 23 (1986).

26 H.R. Brand, Phys.Rev. A33, 643 (1986).

27 H.R. Brand and H. Pleiner, J.Phys.Lett.(Paris) 46, L 711 (1985).

28 H. Pleiner and H.R. Brand, Phys.Rev. A39, 1563 (1989).

29 H.R. Brand and K. Kawasaki, J.Phys.II 2, 1789 (1992).

30 M. Kléman, Rep.Progr.Phys. 52, 1455 (1989).

31 H. Pleiner and H.R. Brand, Phys.Rev.Lett. 54, 1817 (1985).

32 H. Pleiner, Liq.Cryst. 1, 197 (1986).

33 H. Pleiner, Liq.Cryst. 3, 249 (1987).

34 H. Pleiner. Phys.Rev. A37, 3986 (1988).

35 H.R. Brand, P.E. Cladis and H. Pleiner, Macromol. 25, 7223 (1992).

36 H. Pleiner and H.R. Brand, Mol.Cryst.Liq. Cryst. 199, 407 (1991).

37 H. Pleiner and H.R. Brand, Macromol. 25, 895 (1992).

38 H.R. Brand and H. Pleiner, Physica A 208, 359 (1994).

39 H. Pleiner and H.R. Brand in Pattern Formation in Liquid Crystals, ed. by A. Buka and L. Kramer, Springer, Berlin (1995).

40 In this respect we have changed the point of view expressed in H.R. Brand, H. Pleiner and W. Renz, J.Phys.(Paris) 51, 1065 (1990).

41 L.D. Landau and E.M. Lifshitz, Fluid Mechanics, Pergamon Press, New York (1982).

42 S.R. deGroot and P. Mazur, Nonequilibrium Thermodynamics, 2nd ed., Dover, New York (1984).

43 E.H. Lucassen and J. Lucassen, Adv.Colloid Interface Sci. 2, 347 (1969).

44 L. Kramer, J.Chem.Phys. 55, 2097 (1971).

45 D. Langevin, J. Meunier, and D. Chatenay, in Surfactants in Solution Vol.3, and references therein.

46 J.L. Harden and H. Pleiner, Phys.Rev. E49, 1411 (1994).

47 S. Hård and R.D. Neuman, J.Colloid Interface Sci. 115, 73 (1987).

48 H. Yu in Physics of Polymer Surfaces and Interfaces, ed. by I.C. Sanchez, Butterworth, Boston (1992).

49 B.H. Cao and M.W. Kim, Europhys.Lett. 29, 555 (1995). 